\section{Directly modulated cable television transport systems using negative dispersion fiber}

\author{
Hai-Han Lu, MEMBER SPIE \\ National Taipei University of Technology \\ Institute of Electro-Optical Engineering \\ 1, Section 3, Chung-Hsiao East Road \\ Taipei, 10608, Taiwan \\ E-mail: hhlu@ntut.edu.tw \\ Je-Wei Liaw \\ Yi-Shiuan Lee \\ Wan-Lin Tsai \\ Yu-Jie Ji \\ National Taipei University of Technology \\ Department of Electrical Engineering \\ Taipei, 10608, Taiwan
}

\begin{abstract}
A directly modulated AM-VSB cable-television transport system using negative dispersion fiber (NDF) as the transmission medium is proposed and successfully demonstrated. Good performances of carrier-to-noise radio, composite second order, and composite triple beat were obtained over a 70-km NDF transport without optical amplification. The directly modulated laser has a positive chirp, while NDF has a negative dispersion property in the transmission fiber. This negative dispersion property compensates for the laser chirp and results in a system with better transmission performance. (c) 2005 Society of Photo-Optical Instrumentation Engineers. [DOI: 10.1117/1.1869506]
\end{abstract}

Subject terms: directly modulated laser; frequency chirp; negative dispersion fiber.

Paper L040280 received May 23, 2004; revised manuscript received Dec. 1, 2004; accepted for publication Dec. 7, 2004; appeared online Dec. 13, 2004; published online Mar. 4, 2005.

\section{Introduction}

To extend transmission distance is the goal of fiber optical cable television (CATV) transport systems. If the fiber transmission length exceeds several tens of kilometers, a dispersion effect can cause intolerable amounts of composite second order (CSO) and composite triple beat (CTB) distortions. It is necessary to use dispersion compensation devices, such as a chirped fiber grating or a dispersion compensation fiber, ${ }^{1,2}$ to overcome the dispersion effect and consequently decrease the nonlinear distortion. But dispersion compensation devices increase the cost and complexity of systems. Recently, a directly modulated transmitter has become attractive for lightwave transport systems, because of its lower costs compared to an externally modulated transmitter. A directly modulated transmitter, however, has a large chirp which limits the transmission distance due to a small dispersion tolerance. In previous studies, negative dispersion fiber (NDF) has been used as the transmission medium in digital lightwave transport systems,${ }^{3-5}$ but its application in analog lightwave transport

0091-3286/2005/\$22.00 @ 2005 SPIE systems has not been addressed. NDF, which has negative dispersion, is expected to compensate positive laser chirp and improve the dispersion tolerance in a directly modulated CATV transport system. In this work, the architecture of a directly modulated CATV transport system over NDF links is proposed. We successfully demonstrate that directly modulated CATV signals can be transmitted up to a maximum of $70 \mathrm{~km}$ of NDF without optical amplification.

\section{Experimental Setup}

The experimental system configuration of our proposed directly modulated CATV transport systems using NDF as the transmission medium is shown in Fig. 1. Radiofrequency carriers generated from a 77-channel $(\mathrm{CH} 2-78)$ NTSC Matrix SX-16 signal generator are fed into a directly modulated transmitter with a central wavelength of 1550.5 $\mathrm{nm}$ and an optical modulation index (OMI) of $\sim 3.8 \%$ per channel. The system is linked with different transmission lengths of 0 to $70 \mathrm{~km} \mathrm{NDF}$, with an attenuation of 0.212 $\mathrm{dB} / \mathrm{km}$ and a negative dispersion of $-2.5 \mathrm{ps} / \mathrm{nm} / \mathrm{km}$. In order to transmit the optical signal up to $70 \mathrm{~km}$ without optical amplification, the distributed feedback (DFB) laser diode has a high power level of $16 \mathrm{dBm}$. After fiber transmission, the received optical signal was received by an optical receiver. All CATV rf parameters were measured using an HP-8591C CATV analyzer at various lengths of NDF (0 to $70 \mathrm{~km})$.

\section{Experimental Results and Discussions}

In a direct modulation scheme incorporating a DFB laser diode with positive chirp parameter $\Delta v$, the second order harmonic distortion to carrier ratio $\left(\mathrm{HD}_{2} / \mathrm{C}\right)$ and third order intermodulation distortion to carrier ratio $\left(I M D_{3} / C\right)$ can be expressed as ${ }^{6}$

$$
\begin{aligned}
& \frac{H D_{2}}{C}=\frac{1}{4} m \ddot{\beta} z \Omega \sqrt{(4 \cdot \Delta v)^{2}+\left(\ddot{\beta} z \Omega^{3}\right)^{2}} \\
& \frac{I M D_{3}}{C}=-\frac{9}{32}(m \ddot{\beta} z \Omega)^{2}\left[4 \cdot(\Delta v)^{2}+\Omega^{2}\right]
\end{aligned}
$$

where $m$ is the OMI, $\ddot{\beta}$ is the second order dispersion coefficient, $z$ is the fiber transmission length, and $\Omega$ is the $\mathrm{rf}$ signal carrier frequency. A direct way to reduce the positive chirp is to introduce a negative chirp parameter into Eqs. (1) and (2). After employing NDF as the transmission fiber, then Eqs. (1) and (2) can be changed into:

$$
\begin{aligned}
& \frac{H D_{2}}{C}=\frac{1}{4} m \ddot{\beta} z \Omega \sqrt{\left[4 \cdot\left(\Delta v+\beta_{\mathrm{NDF}}\right)\right]^{2}+\left(\ddot{\beta}_{z} \Omega^{3}\right)^{2}} \\
& \frac{I M D_{3}}{C}=-\frac{9}{32}(m \ddot{\beta} z \Omega)^{2}\left[4 \cdot\left(\Delta v+\beta_{\mathrm{NDF}}\right)^{2}+\Omega^{2}\right]
\end{aligned}
$$

where $\beta_{\mathrm{NDF}}$ is the negative dispersion parameter due to NDF. It indicates that the lowest $H D_{2} / C$ and $I M D_{3} / C$ values can be achieved when $\left(\Delta v+\beta_{\mathrm{NDF}}\right)^{2}$ reaches the lowest value which results in better transmission performance. At a fiber length of $60 \mathrm{~km}$, the measured carrier-tonoise radio (CNR), CSO, and CTB values under the NTSC channel number are plotted in Fig. 2. Excellent performances of $\mathrm{CNR} / \mathrm{CSO} / \mathrm{CTB}(>50 / 67.5 / 66 \mathrm{~dB})$ were achieved without optical amplification. 


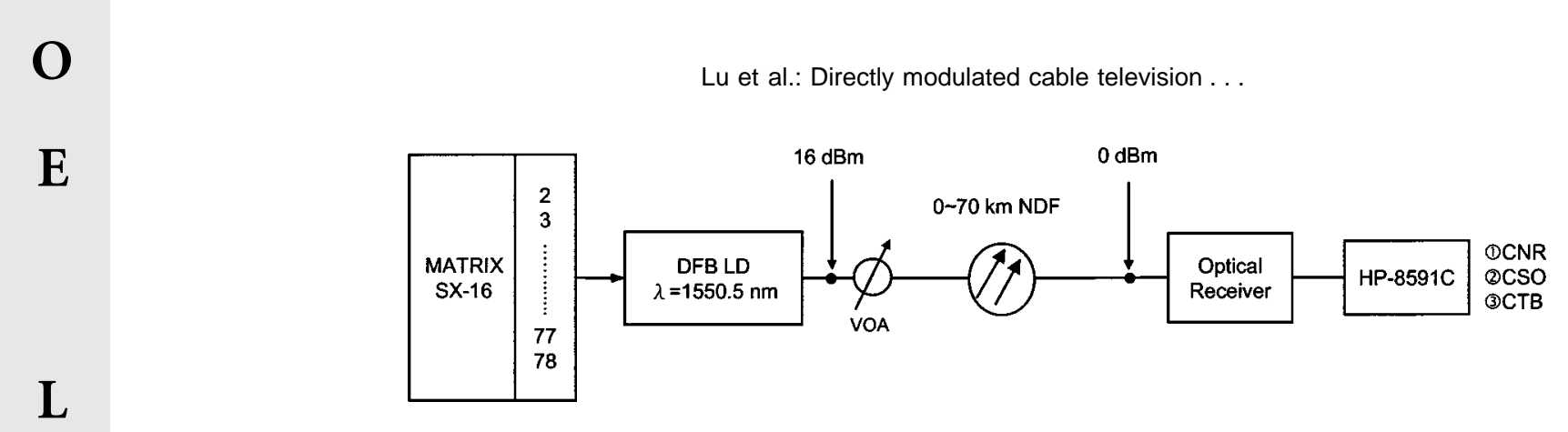

Fig. 1 Experimental system configuration of our proposed directly modulated CATV transport system.

E

$\mathbf{T}$

$\mathbf{T}$

E

$\mathbf{R}$

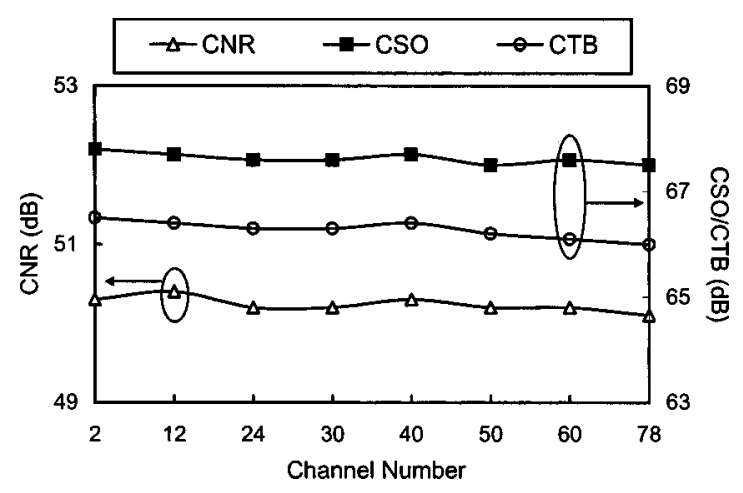

Fig. 2 The measured CNR, CSO, and CTB values under NTSC channel number over a $60-\mathrm{km}$ NDF transport.

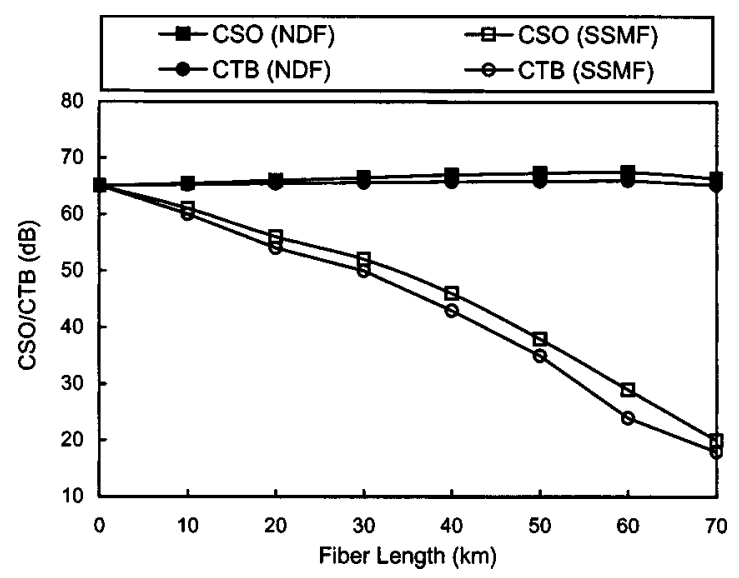

Fig. 3 The measured CSO/CTB values at the highest channel (CH78, worst case) as a function of fiber length.

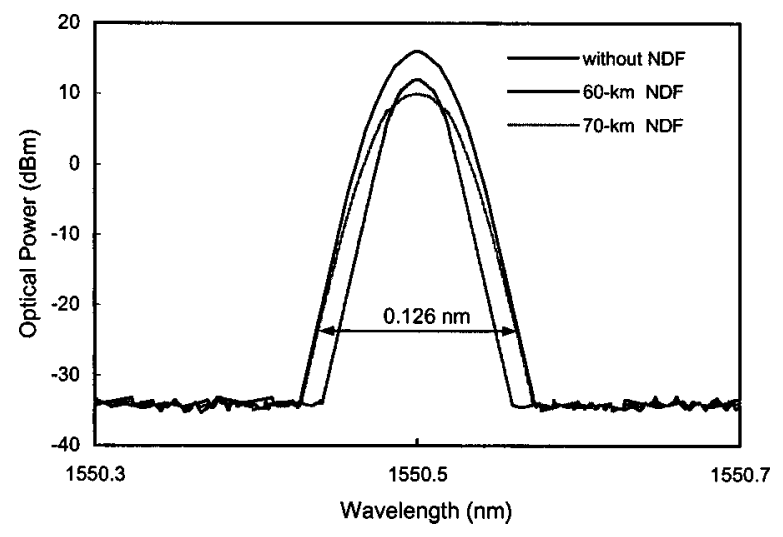

Fig. 4 The optical spectra for a DFB laser diode with/without NDF transport.
The measured $\mathrm{CSO} / \mathrm{CTB}$ values at the highest channel ( $\mathrm{CH} 78$, worst case) as a function of fiber length is shown in Fig. 3. Due to the effect of the accumulated negative dispersion, it is apparent that $\mathrm{CSO} / \mathrm{CTB}$ values improve as the fiber length increases up to $60 \mathrm{~km}$. However, CSO/CTB performances are degraded when the transmission length is more than $60 \mathrm{~km}$. Therefore, it can be concluded that a large amount of negative dispersion will degrade system transmission performance and limit the transmission distance. When the fiber transmission length is $70 \mathrm{~km}$, the measured CSO/CTB values are 66.4/65.2 dB, and both still meet the fiber optical CATV demands $(>65 / 65 \mathrm{~dB})$. In order to make clear the improvement achieved with our proposed setup, a comparison with a standard single-mode fiber (SSMF) transport system is also presented in Fig. 3. When we use SSMF, large amounts of CSO/CTB distortions are generated as the transmission length is increased. The optical spectra for a DFB laser diode with/without NDF transport are shown in Fig. 4. It can be seen that originally the optical signal possesses a wide $0.126-\mathrm{nm}$ spectral linewidth (1550.563 to $1550.437 \mathrm{~nm})$. Over a 60-km NDF transport, the optical signal possesses a narrow 0.098-nm spectral linewidth; over a 70-km NDF transport, the optical signal possesses a $0.124-\mathrm{nm}$ spectral linewidth.

\section{Conclusion}

We successfully demonstrated a directly modulated CATV transport system over $70 \mathrm{~km}$ of NDF without optical amplification. Good performances of CNR, CSO, and CTB were obtained in our proposed systems. Thus, it is possible to implement a cost-effective CATV transport system employing a directly modulated laser and NDF.

\section{References}

1. H. H. Lu, C. T. Lee, and C. J. Wang, "Dispersion compensation in externally modulated transport system using chirped fiber grating as well as large effective area fiber," Opt. Eng. 40(5), 656-657 (2001).

2. D. Piehler, C. Y. Kuo, J. Kleefeld, and C. Gall, "Nonlinear intermodulation distortion in an optically amplified analog video transport system with dispersion compensation fiber," in Proc. Eur. Conf. Opt. Commun. (ECOC'96), WeP.02, 3217-3220 (1996).

3. H. S. Chung, Y. G. Jang, and Y. C. Chung, "Directly modulated 10$\mathrm{Gb} / \mathrm{s}$ signal transmission over $320 \mathrm{~km}$ of negative dispersion fiber for regional metro network," IEEE Photonics Technol. Lett. 15(9), 13061308 (2003).

4. J. A. P. Morgado and A. V. T. Cartaxo, "Directly modulated laser parameters optimization for metropolitan area networks utilizing negative dispersion fibers," IEEE J. Sel. Top. Quantum Electron. 9(5), 1315-1324 (2003).

5. I. Tomkos, B. Hallock, I. Roudas, R. Hesse, A. Boskovic, J. Nakano, and R. Vodhanel, " $10-\mathrm{Gb} / \mathrm{s}$ transmission of $1.55-\mu \mathrm{m}$ directly modulated signal over $100 \mathrm{~km}$ of negative dispersion fiber," IEEE Photonics Technol. Lett. 13(7), 735-737 (2001).

6. M. R. Philips, T. E. Darcie, D. Marcuse, G. E. Bodeep, and N. J. Frigo, "Nonlinear distortion generated by dispersive transmission of chirped intensity-modulated signals," IEEE Photonics Technol. Lett. 3(5), 481-483 (1991). 